\title{
Development of an Efficient Conversion System for GML Documents
}

\author{
Dong-Suk Hong, Hong-Koo Kang, Dong-Oh Kim, and Ki-Joon Han \\ School of Computer Science \& Engineering, Konkuk University, \\ 1, Hwayang-Dong, Gwangjin-Gu, Seoul 143-701, Korea \\ \{dshong, hkkang, dokim, kjhan\}@db.konkuk.ac.kr
}

\begin{abstract}
In this paper, we designed and implemented a Conversion System for GML documents, and evaluated the performance of the system. The conversion system that uses the BXML-based binary encoding specification can directly display geographic information from BXML documents and can convert GML documents to BXML documents without loss of information, and vice versa. BXML is generally more effective in scanning cost and space requirement than GML and coordinate values in the form of BXML can be used directly without conversion.
\end{abstract}

Keywords: Conversion System, GML, BXML, OGC, Geographic Information.

\section{Introduction}

Recently, OGC(Open GIS Consortium) has presented the GML(Geography Markup Language) specification[3,4] for the interoperability of the geographic information. However, since the GML documents are encoded in text and its tag is very repetitive, it yields problems such as large data size, slow transmission time, and enormous document scanning cost[6]. The major method to reduce the size of the text includes compressing such as GZIP[1]. However, as the data compressed by GZIP must be decompressed into the original GML document, the document scanning cost and the coordinate-value converting cost increase enormously.

OGC has proposed the BXML(Binary-XML) encoding specification[5] that can encode the GML document into binary XML format and reduce the document size by removing the repetitive tag names and attributes. And, the BXML encoding specification also can reduce the coordinate-value converting cost to display geographic information by encoding coordinate values into binary values.

In this paper, we designed and implemented a Conversion System for GML documents. The system uses the BXML-based binary encoding specification proposed by OGC. BXML documents are generally more effective in scanning cost and space requirement than GML documents. Especially, coordinate values in the form of BXML documents can be used directly without conversion. The system can directly display geographic information from BXML documents and can convert GML documents to BXML documents without loss of information, and vice versa. In addition, this paper analyzed the performance results of the system. 


\section{Related Works}

OGC proposed the GML specification for interoperability[3,4]. GML is an XML encoding in compliance with ISO 19118 for the transport and storage of geographic information modeled according to the conceptual modeling framework used in the ISO 19100 series and including both the spatial and non-spatial properties of geographic features[2,3]. GML as presently encoded using plain-text XML[7] has three major performance problems[5]: the text in the XML structure is highly redundant and bulky, making it slow to transfer over the Internet; the lexical scanning of XML is unexpectedly costly; and the conversion of text-encoded numerical coordinate and observation values is also very costly.

BXML was proposed for more effective transmission by reducing the size of the XML document without changes in contents for a limited environment[5]. If an XML file is used as a database of objects with a specific attribute used as a primary key, then it will be greatly more efficient to randomly seek directly to the data tokens that encode those objects. BXML can directly represent raw binary data without any indirect textual-encoding methods, and a backward-compatibility mechanism is provided to enable the translation raw-binary blocks into an equivalent XML representation.

\section{System Development}

Fig. 1 shows the architecture of the conversion system for GML documents presented in this paper.

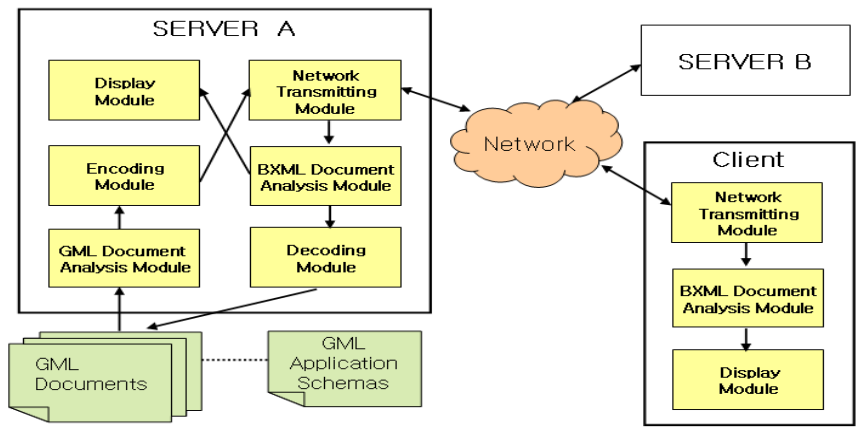

Fig. 1. System Architecture

This system is divided into a server and a client. The server system is composed of a GML document analysis module to analyze GML documents, an encoding module to encode GML documents into BXML documents, a BXML document analysis module to analyze BXML documents, a decoding module to decode BXML documents into GML documents, a display module to read the spatial data from BXML documents for display, and a network transmitting module to transmit the data encoded into BXML documents through network. The client system is composed of a network transmitting module, a BXML document analysis module, and a display module. 
In order to convert the GML document into the BXML document, the GML document is analyzed, made into tokens, and encoded into the corresponding binary values based on the code values. Fig. 2 and Fig. 3 shows an example of a GML document and a BXML document converted from the GML document of Fig. 2, respectively. In addition, In order to convert the BXML document into the original GML document, the BXML document is analyzed and the token types are classified.

The display module derives out the spatial data from a BXML document and displays it on the screen as maps. Fig. 4 shows the screen display from the BXML document of Fig. 3.

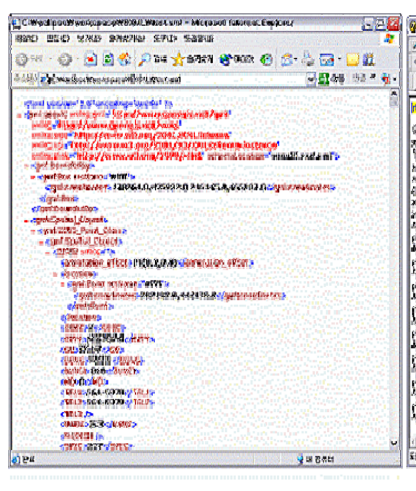

Fig. 2. GML Document

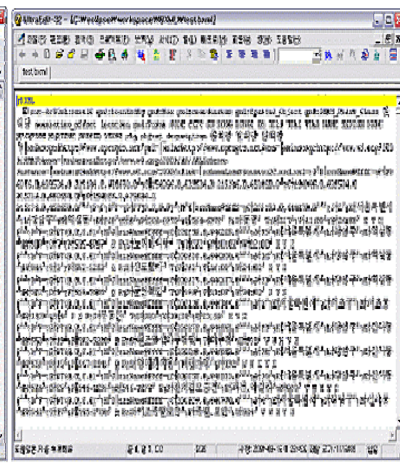

Fig. 3. BXML Document

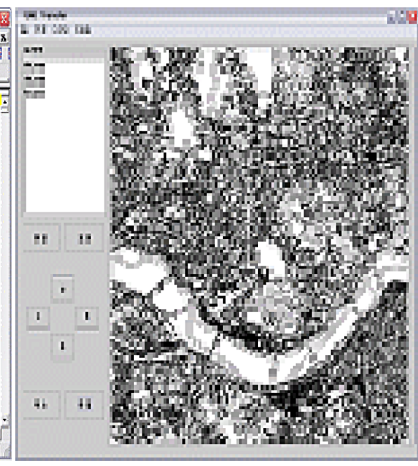

Fig. 4. Screen Display

\section{Performance Evaluation}

This chapter examines the performance evaluation results of the conversion system for GML documents. The GML documents used are 20Mbytes, 40Mbytes, 60Mbytes, and $80 \mathrm{Mbytes}$ and are composed of point data, polyline data, and polygon data.

Fig. 5 illustrates the document size when the GML documents are compressed by GZIP and when the GML documents are encoded into the BXML documents. As shown in Fig 5, the compression of the GML document using GZIP achieves the better compression rate. However, the GZIP compression does not consider document scanning but solely reduces the data size. Therefore, the compressed GZIP document should be decompressed and restored to its original state in order for us to use the data contained within. On the other hand, encoding into the BXML document can save more document scanning cost than the GZIP compression.

Fig. 6 illustrates the display time of the geographic information of the GML document and the BXML document. As shown in Fig 6, using the BXML document rather than the GML document achieves faster display time and the gap between the two document types increases as the file size enlarges. In case of the GML document, since all data are encoded in texts, the coordinate values must be separated for coordinate value conversion. However, in case of the BXML document, since its geographic data are encoded in its original data type, faster speed is possible as it can be used without any type conversion process. 


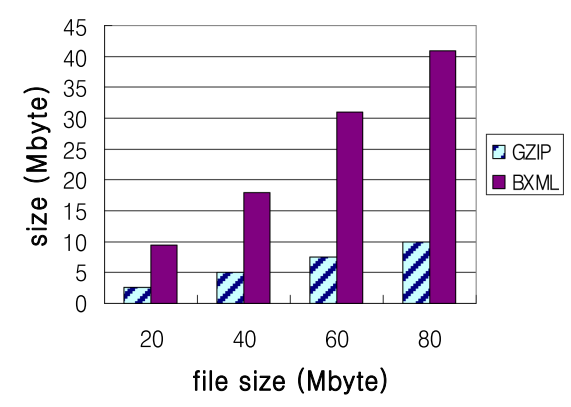

Fig. 5. Comparison of Compression Size

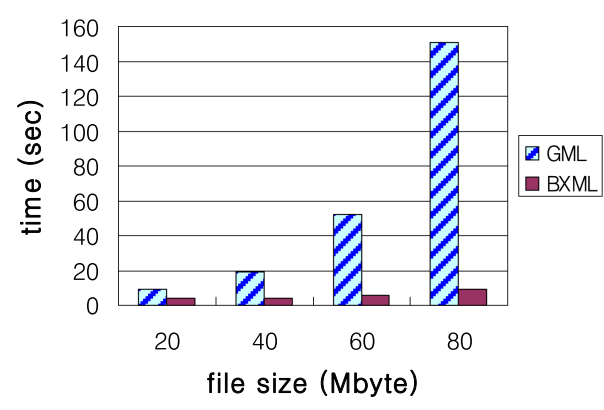

Fig. 6. Comparison of Display Time

\section{Conclusion}

This paper designed and implemented the conversion system for GML documents that can convert GML documents into BXML documents without loss of information, and vice versa. The system also can directly display geographic information from the BXML documents. By using the conversion system, it is possible to enhance the transmission speed by reducing the GML document size and also can enhance the document scanning speed for faster display of the geographic information of the BXML documents.

\section{Acknowledgements}

This research was supported by the Seoul Metropolitan Government, Korea, under the Seoul R\&BD Program supervised by the Seoul Development Institute.

\section{References}

1. IETF RFC 1952: GZIP File Format Specification Version 4.3, http://www.ietf.org/ rfc/rfc1592.txt (1996).

2. ISO/TC 211: ISO 19136 Geographic Information - Geography Markup Language(GML) (2004).

3. OpenGIS Consortium, Inc.: Geography Markup Language(GML) Implementation Specification 3.1.0. (2004).

4. OpenGIS Consortium, Inc.: Geography Markup Language(GML) Version 3.1.1 Schemas (2005).

5. OpenGIS Consortium, Inc.: Binary-XML Encoding Specification 0.0.8. (2003).

6. Piras, A., Demontis, R., Vita, E. D., Sanna, S.: Compact GML: Merging Mobile Computing and Mobile Cartography, Proceedings of the 3rd GML and Geo-Spatial Web Service Conference (2004).

7. W3Consortium: Extensible Markup Language (XML) 1.0., (Second Edition), http://www.w3.org/ TR/REC-xml (2000). 